\title{
A!
}

This is an electronic reprint of the original article.

This reprint may differ from the original in pagination and typographic detail.

Götz, Georg; Schlecht, Sebastian J.; Pulkki, Ville

\section{A dataset of higher-order Ambisonic room impulse responses and 3D models measured in a room with varying furniture}

Published in:

2021 Immersive and 3D Audio: from Architecture to Automotive (I3DA)

DOI:

10.1109/I3DA48870.2021.9610933

Published: 23/09/2021

Document Version

Peer reviewed version

Please cite the original version:

Götz, G., Schlecht, S. J., \& Pulkki, V. (2021). A dataset of higher-order Ambisonic room impulse responses and $3 D$ models measured in a room with varying furniture. In 2021 Immersive and 3D Audio: from Architecture to Automotive (I3DA) (pp. 1-8). [9610933] IEEE. https://doi.org/10.1109/I3DA48870.2021.9610933

This material is protected by copyright and other intellectual property rights, and duplication or sale of all or part of any of the repository collections is not permitted, except that material may be duplicated by you for your research use or educational purposes in electronic or print form. You must obtain permission for any other use. Electronic or print copies may not be offered, whether for sale or otherwise to anyone who is not an authorised user. 


\title{
A dataset of higher-order Ambisonic room impulse responses and 3D models measured in a room with varying furniture
}

\author{
Georg Götz ${ }^{1 *}$, Sebastian J. Schlecht ${ }^{1,2}$, and Ville Pullki ${ }^{1}$ \\ ${ }^{1}$ Aalto Acoustics Lab, Department of Signal Processing and Acoustics \\ ${ }^{2}$ Media Lab, Department of Media \\ Aalto University, Espoo, Finland \\ *Email: georg.gotz@aalto.fi
}

\begin{abstract}
This paper presents Motus, a new dataset of higherorder Ambisonic room impulse responses. The measurements took place in a single room while varying the amount and placement of furniture. 830 different room configurations were measured with four source-to-receiver configurations, resulting in 3320 room impulse responses in total. The dataset features various furniture object placements, including non-uniform distributions of absorptive material and cases with occluded direct paths between source and receiver. All acoustic measurements are accompanied by matching $3 \mathrm{D}$ models and $360^{\circ}$-photographs of the room. After describing the dataset, we demonstrate its usage with a reverberation time analysis. The analysis reveals that most of our measurements follow the expected relationship between absorption area and reverberation time. Some exceptional cases feature particular room acoustic phenomena, such as non-uniform absorption area distributions or multi-slope decays. Additionally, we show with a large number of measurements that furniture placement can significantly affect the reverberation time of a room. The dataset can be used to investigate room acoustic topics such as the acoustic effects of absorber placements or the decay behavior of rooms.

Index Terms-Room impulse response, dataset, acoustic mea-
\end{abstract} surement, varying furniture, room acoustics, virtual reality

\section{INTRODUCTION}

Room geometry, furnishing, and the acoustic treatment of walls have a considerable effect on how sound propagates inside a space [1]-[4]. These changes to the physical sound-field also affect how sound inside a room is perceived. For example, room acoustic considerations are crucial for facilitating good speech intelligibility [5].

The sound-field in a room can be captured using room impulse responses (RIRs), thus representing the acoustical phenomena between a specific source and receiver position. Microphone arrays can simultaneously measure multiple RIRs at spatially distributed receiver positions. Such microphone array recordings can then be encoded into higher-order Ambisonic room impulse responses (HOA-RIRs) [6]. They are a versatile way of describing the sound-field of a room because they include additional spatial information about the soundfield that cannot be represented by a single-channel RIR. They are the basis for research on spatial audio capture and reproduction [7], [8]. Moreover, they have also been bene- ficially used in recent sound-field analysis and visualization approaches [9], [10].

\section{A. Prior Work}

Many RIR datasets are publicly available now. They differ regarding the number of measured source and receiver positions, the resolution of their spatial distribution on a measurement grid, the number of measured acoustic environments, the utilized microphones (omnidirectional, binaural, or microphone array), and the utilized sound sources. Datasets that include many acoustic environments have traditionally been of interest for the development and evaluation of dereverberation and source separation algorithms. Moreover, such datasets are also appealing for room acoustic research or the development of room simulation algorithms to obtain generalizable results. The following section summarizes some notable datasets that contain RIR measurements in various acoustic environments.

In [11], a dataset of microphone array RIRs was collected to aid in developing and evaluating source separation algorithms. The dataset includes different source-to-receiver combinations in a varechoic room, measured at three different reverberation times between $160 \mathrm{~ms}$ and $610 \mathrm{~ms}$. In [12], RIRs were measured in three rooms of considerably different volumes, using an omnidirectional and a first-order Ambisonic microphone. The dataset aimed to cover big areas with dense grids of measurements, and therefore it contains over 700 RIRs at 130 different receiver positions, while the source position was kept static. In [13], a large set of RIRs was measured in four different rooms of various volumes. It was intended to be used for room-acoustic analysis and to develop and evaluate spatial audio technology. The dataset includes RIR measurements conducted with various microphones, which were moved on a microscopic scale with a robot arm. In addition, the dataset contains basic 3D models and spherical photographs of the measured rooms. Other RIR datasets of variable acoustic conditions were collected for the development and evaluation of dereverberation algorithms [14], [15], for hearing-aid research [16], or for auralization [17], [18]. 


\section{B. Outline}

This paper presents the Motus dataset. It is a new dataset of higher-order Ambisonic RIRs, which differs from previous ones regarding three aspects. Firstly, the present dataset includes complete descriptions of every measured room configuration in terms of $3 \mathrm{D}$ models and supplementary $360^{\circ}$ photographs. Secondly, with 830 different acoustic conditions, the present dataset features a bigger acoustic variety than previous datasets, exhibiting reverberation times between $0.5 \mathrm{~s}$ and $2 \mathrm{~s}$ at $1 \mathrm{kHz}$. Thirdly, as opposed to the datasets collected in varechoic rooms, the different acoustic conditions in the present dataset are realized by varying the quantity and placement of the furniture. Therefore, the present dataset also features complex geometries and acoustic wave phenomena like scattering.

The dataset may be valuable for future room acoustic research. For example, it can be used to study the decay behavior of rooms or the acoustic effects of absorber placements. Such analysis follows the motivation of previous studies, which have for example investigated the influence of sound-absorbing material placement on reverberation time [1], [2] and other parameters like clarity (C50) or the speech transmission index (STI) [1]. Future research could benefit from the present dataset, because some furniture quantities have been measured with 40 to 120 different spatial distributions, whereas the above studies were limited to at most 10 absorber placements. The large number of measurements will be beneficial for using data-driven machine-learning approaches while investigating the above research topics.

The remainder of this paper is organized as follows. Section II describes the data acquisition process and the measurement technique. Section III presents the dataset organization and its storage formats. Section IV explores the dataset content in more detail and demonstrates its usage with a reverberation time analysis of the measurements. Section V summarizes our findings and concludes the paper.

\section{DATASET ACQuisition}

The Motus dataset is a new dataset of higher-order Ambisonic RIRs, which was measured using an em32 Eigenmike microphone array and Genelec 1030A loudspeakers. Logarithmic sine sweeps [19] with a length of $5 \mathrm{~s}$ were used as excitation signals. All RIRs were measured in a single $4.9 \mathrm{~m} \times 4.4 \mathrm{~m} \times 2.9 \mathrm{~m}$ (approx. volume of $60 \mathrm{~m}^{3}$ ) seminar room of Aalto University. The dataset contains RIR measurements from four different source-to-receiver configurations. They are depicted in Figure 1 and remained static throughout the dataset collection process. Additionally, a spherical camera was placed above the microphone array to capture $360^{\circ}$-photographs of every measurement.

The measurement room has concrete walls, a vinyl-coated concrete floor, a single door, and two large windows. Variation was introduced into the dataset by changing the number of furniture objects and their position and orientation. Table I summarizes the different furniture objects used for the measurements and their available total quantities.

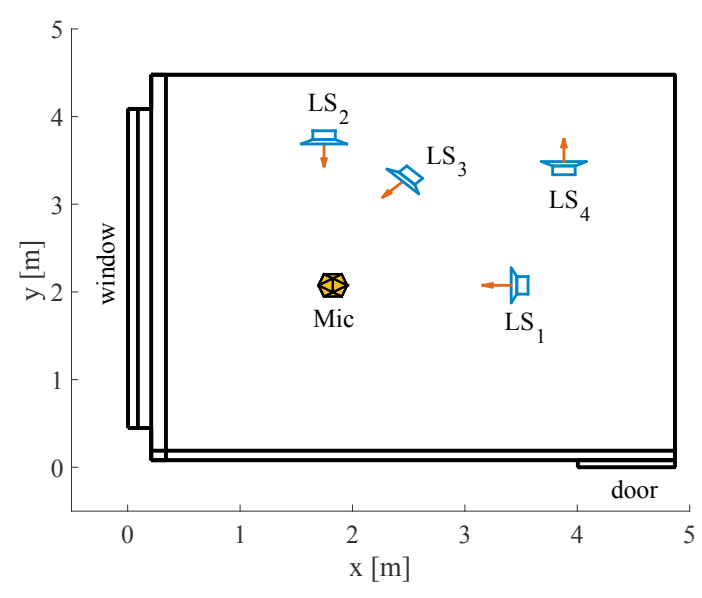

Fig. 1. Floor plan of the seminar room in which the dataset was collected. The microphone and loudspeaker positions remained static throughout the dataset collection.

TABLE I

FURNITURE OBJECTS USED DURING THE MEASUREMENTS OF THE DATA SET.

\begin{tabular}{llc}
\hline Furniture object & Description & Available quantity \\
\hline Shelf & Rollable bookshelf & 6 \\
\hline Drawer & $\begin{array}{l}\text { Rollable drawer, the type, } \\
\text { which is used next to a desk }\end{array}$ & 4 \\
\hline Absorption wedge & $\begin{array}{l}\text { Absorption wedges, the type, } \\
\text { which is used in anechoic } \\
\text { chambers }\end{array}$ & 50 \\
\hline Carpet tile & approx. $0.5 \mathrm{~m} \times 0.5 \mathrm{~m}$ & 56 \\
\hline
\end{tabular}

In the following, we will use the term room configuration to describe unique combinations of furniture quantity, position, and orientation. The present dataset contains 830 room configurations. For each of these room configurations, RIRs were measured with the four source-to-receiver configurations mentioned earlier, thus resulting in 3320 unique RIR measurements in total.

The furniture was mostly moved on the floor and rotated on the horizontal plane to represent common furniture placements. The furniture objects were positioned freely in space, i.e., they could be placed at the walls and at any other position inside the room. In some room configurations, the direct path between the loudspeakers and the microphone array was occluded. Only 10 room configurations featured less than 56 carpet tiles. The carpet tiles remained at the same position for all other room configurations. The absorption wedges were either placed on the floor or on top of the shelves, the drawers, or the window sill. None of the configurations involved an acoustic treatment of the ceiling, such as hanging absorption wedges. Throughout the dataset collection, the number of absorption wedges was varied in increments of 10 between 0 and 50, resulting in six groups. The reasoning for this 
TABLE II

ASSUMED ABSORPTION COEFFICIENTS FOR THE DIFFERENT FURNITURE OBJECTS AND SURFACE MATERIALS IN THE ROOM.

\begin{tabular}{lcccccc}
\hline \multirow{2}{*}{ Furniture object / room surface } & \multicolumn{5}{c}{ Absorption coefficient $\boldsymbol{\alpha}$} \\
\cline { 2 - 7 } & $\mathbf{1 2 5} \mathbf{H z}$ & $\mathbf{2 5 0 ~ H z}$ & $\mathbf{5 0 0 ~} \mathbf{~ z}$ & $\mathbf{1} \mathbf{~ k H z}$ & $\mathbf{2} \mathbf{~ k H z}$ & $\mathbf{4} \mathbf{~ k H z}$ \\
\hline Absorption wedge & 0.25 & 0.50 & 0.85 & 0.95 & 0.90 & 0.90 \\
Shelf, drawer, door & 0.15 & 0.11 & 0.10 & 0.07 & 0.06 & 0.07 \\
Carpet tile & 0.03 & 0.05 & 0.05 & 0.25 & 0.35 & 0.50 \\
Window & 0.30 & 0.20 & 0.10 & 0.07 & 0.05 & 0.02 \\
Floor & 0.02 & 0.02 & 0.03 & 0.04 & 0.04 & 0.05 \\
Walls & 0.02 & 0.03 & 0.03 & 0.03 & 0.04 & 0.07 \\
\hline
\end{tabular}

granularity is twofold. Firstly, investigating the acoustic effects of furniture placement (c.f. Section IV-B) requires room configurations with identical furniture amounts. Secondly, varying the position of the wedges inside the room was more timeefficient than varying their quantity.

To complement the acoustic measurements with information about the room geometry and the furniture placement, we used SketchUp to manually create $3 \mathrm{D}$ models of every room configuration. The resulting 3D models were exported to MATLAB, where they were added as triangulated meshes to the room configuration metadata (c.f. Section III). Additionally, we included the absorption coefficients of the furniture and the room surfaces. The coefficients were obtained from textbook tables. They are summarized in Table II.

\section{DATASET DESCRIPTION}

The Motus dataset consists of higher-order Ambisonic RIRs measured in a single room with 830 different furniture configurations. It is publicly available online [20]. Table III provides an overview of the dataset, its organization into smaller parts, and the available formats. Some additional background information will be given in the following.

Figure 2 shows three exemplary room configurations to illustrate the nature of the dataset. The companion page of this paper $^{1}$ also includes a flip-book-inspired animation, in which we showcase all measured room configurations of the dataset with spherical photographs and 3D models. An exemplary 3D model of the dataset is depicted in Figure 3.

Although the dataset consists of 830 unique room configurations, some of them feature the same amount of furniture objects and therefore differ only with respect to the furniture placement. More precisely, the room configurations of the dataset can be divided into 60 groups, each of which represents the room configurations with a particular amount of furniture objects or absorption area. This grouping effectively quantizes the possible total absorption area values of the entire dataset into 60 non-uniform steps. Figure 4 illustrates the absorption area variability in the dataset due to the different amounts of furniture. Although the figure groups all configurations into equally-sized bins of 1 Sabin, it illustrates how many room configurations fall into the different absorption area ranges included in the dataset. It is important to note that not all furniture object quantities were measured equally often. Room

\footnotetext{
${ }^{1}$ http://research.spa.aalto.fi/publications/papers/i3da21-motus/
}

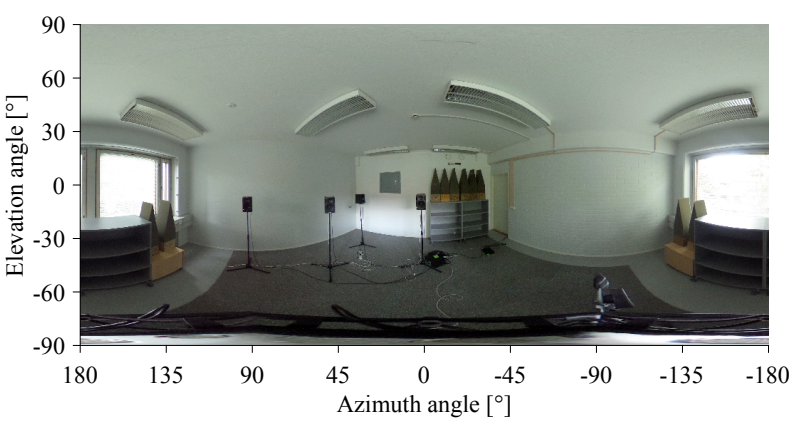

(a) Small amount of furniture (measurement \#769)

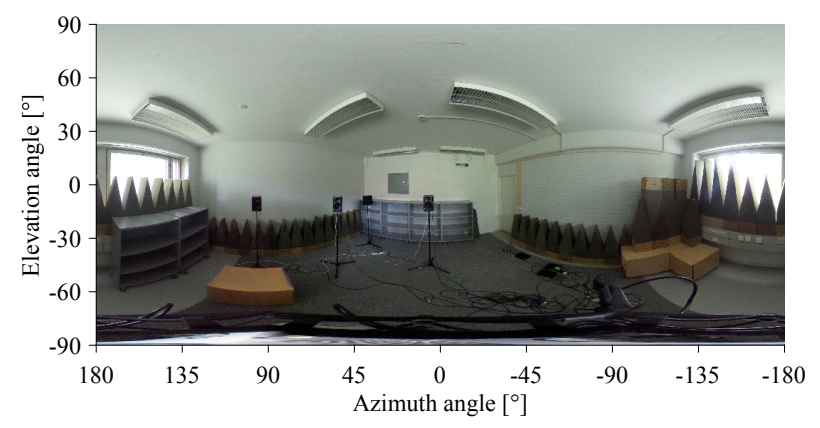

(b) Large amount of furniture (measurement \#20)

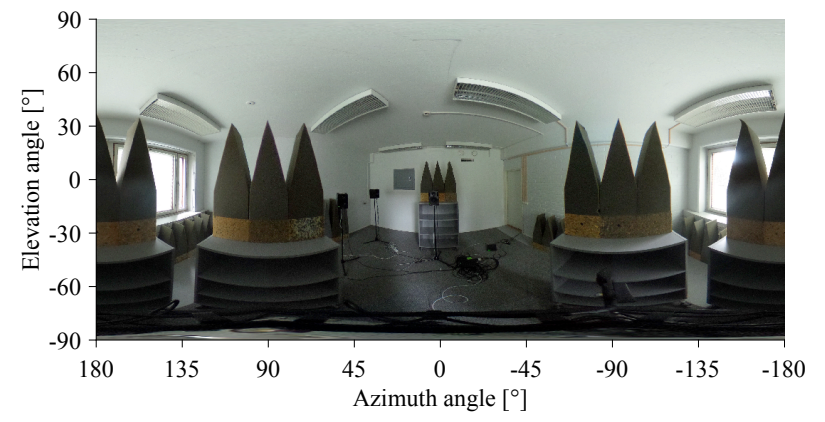

(c) Occluding furniture objects between loudspeaker 2 (azimuth $=90^{\circ}$ ) and the microphone array (measurement \#212)

Fig. 2. Comparison of different measured room configurations. The dataset includes configurations ranging from a relatively empty (2a) to a full room (2b) It also includes configurations in which the direct path between the loudspeakers and the microphone array is occluded $(2 \mathrm{c})$. 
TABLE III

DATASET OVERVIEW, ORGANIZATION, AND FORMAT

\begin{tabular}{|c|c|c|}
\hline Name & Description & Format \\
\hline raw_rirs & Raw Eigenmike RIRs & 32-channel .wav $(48 \mathrm{kHz})$ \\
\hline sh_rirs & 4th-order Ambisonic RIRs & 25-channel .wav ( $48 \mathrm{kHz}, \mathrm{ACN}, \mathrm{SN} 3 \mathrm{D})$ \\
\hline metadata & & .mat and JSON \\
\hline room & $\begin{array}{l}\text { 3D model of room configuration: one model for the entire configuration } \\
\text { including all of the furniture }\end{array}$ & $\begin{array}{l}\text { - vertex list (point cloud) } \\
\text { - triangulated surfaces: connectivity, normal } \\
\text { - for every surface: absorption coefficients } \\
\text { Note: the } 3 D \text { models are also available as fbx files }\end{array}$ \\
\hline furniture_objects & $\begin{array}{l}\text { - Furniture object quantities } \\
\text { - 3D model split according to furniture object types }\end{array}$ & $\begin{array}{l}\text { - furniture object quantities } \\
\text { - vertex list (point cloud) } \\
\text { - triangulated surfaces: connectivity, normal } \\
\text { - for every surface: absorption coefficients }\end{array}$ \\
\hline $\mathrm{rcv}$ & Information about the microphone position and orientation & $3 \mathrm{D}$ vectors (position and orientation) \\
\hline $\operatorname{src} 1, \operatorname{src} 2, \operatorname{src} 3, \operatorname{src} 4$ & Information about the loudspeaker positions and orientations & $3 \mathrm{D}$ vectors (position and orientation) \\
\hline
\end{tabular}

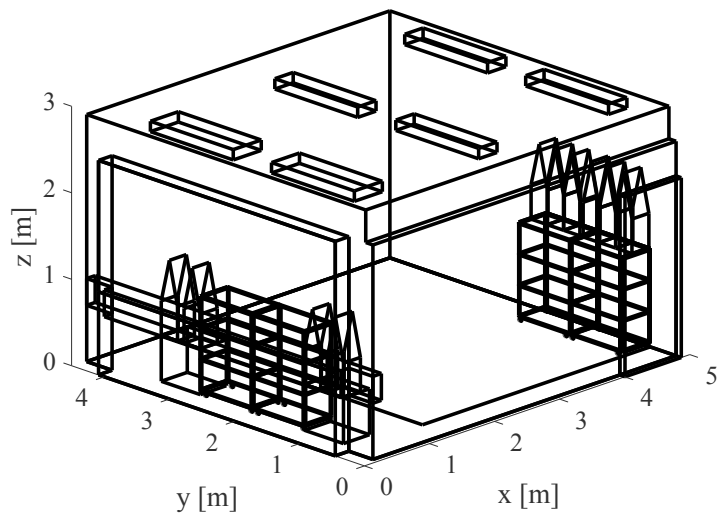

Fig. 3. Exemplary 3D model of a measurement. The depicted room configuration coincides with the spherical photograph of Figure 2a.

configurations with large amounts of furniture were measured more frequently than comparably empty rooms. This can be attributed to the higher number of possibilities to distribute furniture when more objects are available.

Every measurement contains the raw 32-channel Eigenmike RIRs and a corresponding 4th-order Ambisonic representation. Both are stored as multichannel .wav-files with a sampling rate of $48 \mathrm{kHz}$. The 25 components of the 4th-order Ambisonic stream are ordered according to the ACN convention with SN3D normalization [8].

$3 \mathrm{D}$ models are available as .fbx-files for every room configuration. Additionally, the 3D models are part of separate metadata structures. They are available as JSON and .mat-files, thus making them readable with MATLAB or Python. Inside these metadata structures, the 3D models are stored as triangulated meshes, consistent with MATLAB's (triangulated) surface format, such that every mesh consists of a vertex list (i.e., point cloud) and a $\left(N_{\text {surfaces }} \times 3\right)$ connectivity matrix, which describes the vertices of every surface by indexing into the vertex list. Furthermore, surface normals and absorption coefficients are available for every mesh surface.

Additionally, the dataset contains a $360^{\circ}$-photograph of every measured room configuration as seen from the position of the microphone array. The images are available as .mat and .jpeg-files.

Lastly, we provide additional MATLAB scripts that can be used when working with the dataset. For example, we included functions to visualize the $3 \mathrm{D}$ models or the spherical photographs. Furthermore, we provide utility functions that can be used on the metadata structures to extract the overall absorption area or furniture object quantities of a room configuration with a single function call.

\section{REVERBERATION TIME ANALYSis}

In the following section, we will demonstrate applications of the dataset. More precisely, we will start by exploring how the total absorption area varies among the measured room configurations and how the reverberation time changes accordingly. Afterwards, we will use the present dataset for demonstrating how the reverberation times vary when only the furniture placement is changed, while keeping the total absorption area of the room constant. Although the acoustic effect of absorber placement has already been studied before [1], [2], this section outlines how the previous studies can be extended with the large number of measurements of the present dataset.

For all of the following analyses, the reverberation times were determined in octave-bands from the omnidirectional channel of the higher-order Ambisonic RIR measurements by fitting a non-linear decay-plus-noise model according to [21]. The total absorption areas of the room configurations were calculated by summing the absorption areas of all room and furniture object surfaces without considering occluded surfaces. For example, an absorption wedge in a room corner 


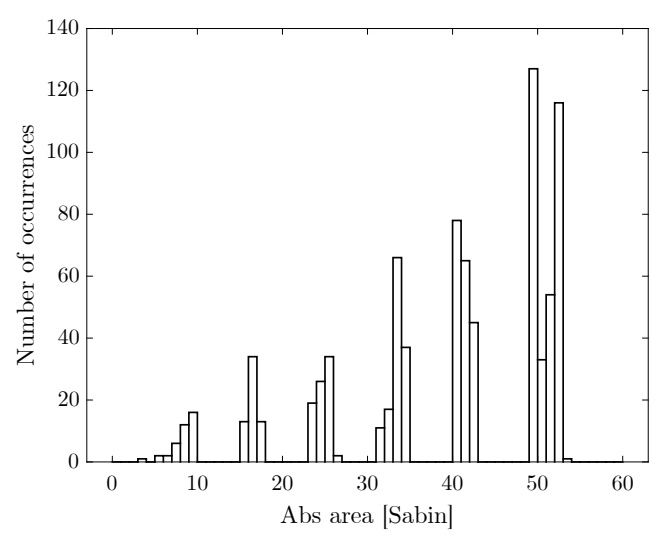

Fig. 4. Histogram depicting the number of measured room configurations for different amounts of total absorption area (at $1 \mathrm{kHz}$ ) in the room. The histogram has a bin-size of 1 Sabin. It is based on the 830 measured room configurations, thus not counting duplicate occurrences due to the 4 sources.

contributes exactly the same absorption area to a room configuration as a wedge inside the room. It should be noted that this constitutes a simplification of our model because, from a physical point of view, some of the wedge's surface areas would not be exposed to sound at the corner position.

\section{A. Reverberation time analysis for varying absorption area}

Due to a large number of different furniture configurations, the dataset exhibits a variety of acoustic conditions. This diversity of the dataset can, for example, be observed when investigating the reverberation times of the different configurations. In acoustical terms, the amount of furniture usually corresponds to the extent of the absorption area. The relationship between the absorption area and the reverberation time of a room is well researched [4]. Typically, the reverberation time decreases with an increasing absorption area. The dataset's absorption area variability due to the different amounts of furniture has already been depicted in Figure 4. Consequently, a considerable variability of reverberation times can be observed in the dataset, and the relationship to the absorption area is in line with the general trend predicted by theory. Figure 5 illustrates this relationship by depicting the total amount of absorption area with the reverberation time. The plots show several noticeable peculiarities.

Firstly, in the frequency bands $500 \mathrm{~Hz}$ and above, a distinct clustering into six groups can be observed. This clustering was caused by the granularity in the number of absorption wedges inside the room. Between these groups, the jump in the amount of absorption area is significant because the wedges are highly absorptive. The granularity in the other furniture object quantities did not cause such a drastic clustering, because their materials are less absorptive. Nevertheless, quantity variations of these objects still caused an inevitable absorption area variability within the groups. The grouping can also be observed in Figure 4.
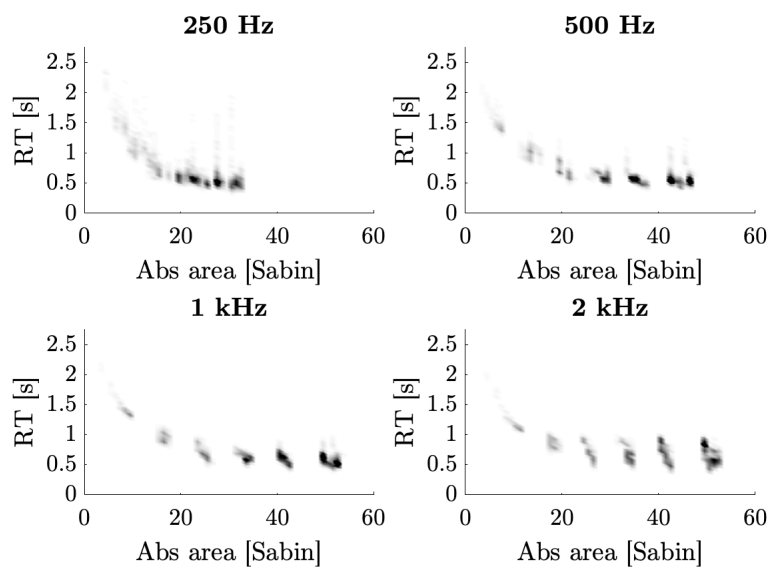

$4 \mathbf{~ k H z}$

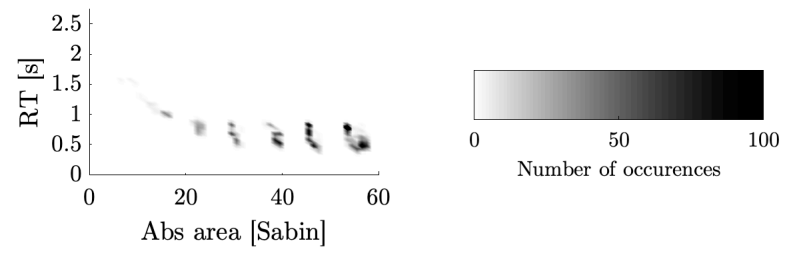

Fig. 5. Top-down view of a 3D-histogram depicting the relationship between total absorption area in the room and reverberation time determined from the room impulse responses. All 3320 RIRs were used for producing this plot. The reverberation time analysis was carried out in octave-bands. The color indicates how often a particular combination of absorption area and reverberation time occurs in the dataset.

Secondly, it may appear unusual that the reverberation time does not decrease below approximately $0.4 \mathrm{~s}$, despite increasing the amount of absorption considerably. However, the absorption was introduced by adding absorption wedges, which have a comparably low total surface area compared to the walls or shelves. Therefore, the average absorption coefficient of the entire room configuration changed only minimally. Another possible explanation for steady reverberation time values at increasing absorption areas would be the lack of diffusion due to non-uniform absorption material distributions. For example, increasing the absorption area in only one corner of the room would not reduce the reverberation time indefinitely. Nevertheless, non-uniform absorption material distributions cannot explain the observed values for all room configurations because the furniture was moved around the entire room, thus creating occasions for scattering in most of the room configurations.

Lastly, in the $250 \mathrm{~Hz}$ frequency band, the figure features some unusually high reverberation time values of up to $2 \mathrm{~s}$ for absorption areas around 30 Sabin. A thorough analysis of the measured configurations revealed that there seem to be two reasons for this observation. Firstly, some of the measurements featured highly non-uniform distributions of absorption area, such as room configurations with all 50 wedges placed in front of a single wall. These cases also resulted in higher reverberation time values than configurations with more equal absorption distributions (c.f. Section IV-B). Secondly, the 


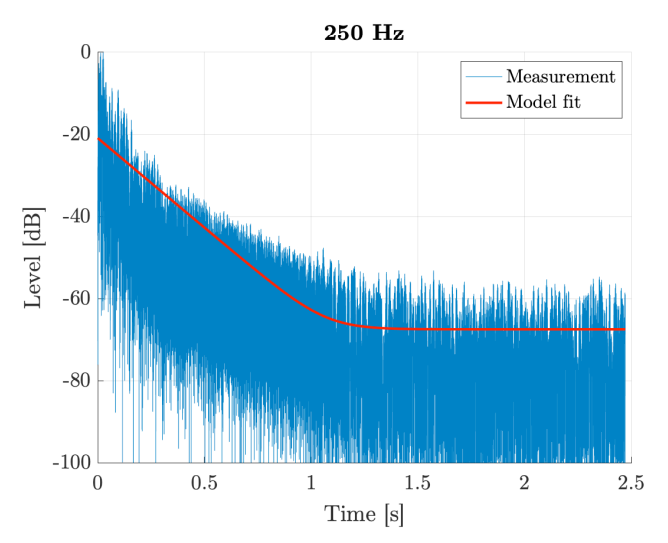

Fig. 6. Some room configurations include double-slope decays. As depicted here for the measurement \#178, standard reverberation time fitting approaches may produce inaccurate results in these cases.

fitting algorithm returned incorrect reverberation times for room configurations that feature double-slope decays. The non-linear decay-plus-noise model, as implemented in [21], fitted neither of the two slopes correctly, thus resulting in an averaged slope somewhere between the two slopes. Figure 6 illustrates this fitting problem with one of the dataset's doubleslope decays. Other toolboxes, such as the ITA toolbox [22] or the IoSR toolbox [23], implement a linear least-squares fitting to decay curves from reverse-time integrated squared impulse responses according to ISO 3382 [24]. These toolboxes offer the possibility to set the fitting range (i.e., $T_{20}, T_{30}$, or $\left.T_{60}\right)$ with a parameter. While an appropriate choice of this parameter may result in a correct fit of the first slope, we did not find a one-fits-all fitting range for determining correct decay slopes of the entire dataset. This observation illustrates that more robust fitting algorithms are required to analyze significant amounts of data. For datasets as big as the presented one, a dedicated fitting algorithm tuning is infeasible or too time-consuming.

\section{B. Reverberation time analysis for constant absorption area}

As the dataset features multiple room configurations, in which the amount of furniture was constant while only the furniture placement was varied, it can be used to analyse room acoustic parameters with the furniture placement as the only independent variable. In this paper, we want to use the present dataset to investigate the effect of different furniture distributions on the reverberation time.

Figure 7 shows the reverberation time variability for the five most frequently occurring furniture combinations of the dataset. The figure illustrates that considerable reverberation time differences of up to $1 \mathrm{~s}$ were observed for room configurations that only differed regarding the furniture placement. Two peculiarities of the figure are noteworthy.

Firstly, exceptionally high reverberation times were observed for the furniture combination $\mathrm{C} 1$ in the $250 \mathrm{~Hz}$ and $500 \mathrm{~Hz}$ frequency bands. An analysis of the corresponding
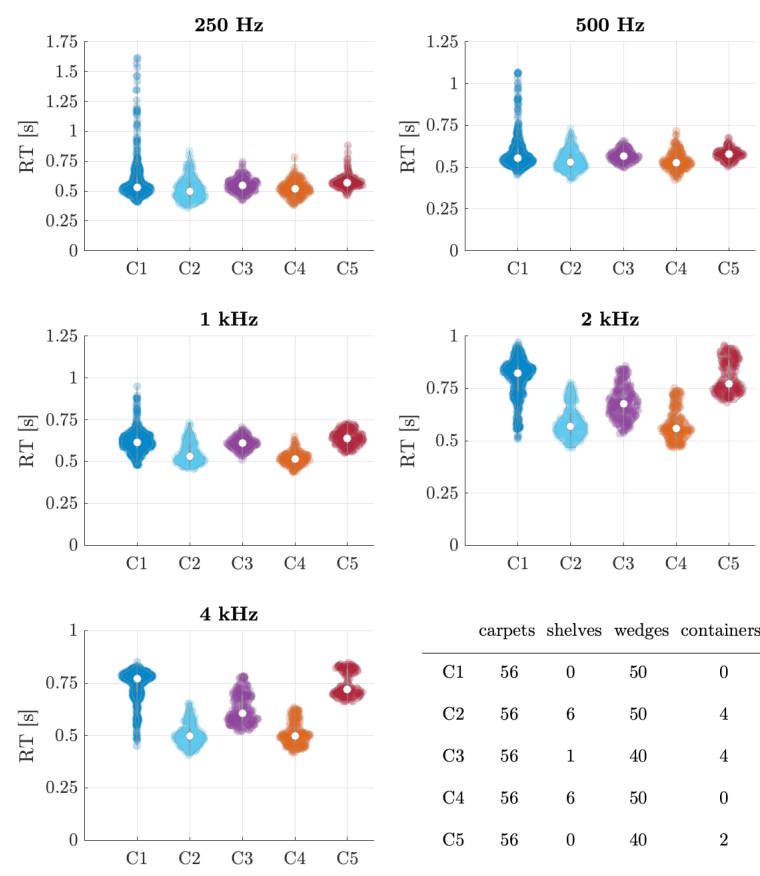

\begin{tabular}{ccccc}
\multicolumn{4}{c}{ carpets shelves } & wedges containers \\
\hline $\mathrm{C} 1$ & 56 & 0 & 50 & 0 \\
$\mathrm{C} 2$ & 56 & 6 & 50 & 4 \\
$\mathrm{C} 3$ & 56 & 1 & 40 & 4 \\
$\mathrm{C} 4$ & 56 & 6 & 50 & 0 \\
$\mathrm{C} 5$ & 56 & 0 & 40 & 2
\end{tabular}

Fig. 7. Variability of reverberation time for room configurations with the same number of furniture objects and hence with the same absorption area. All 3320 RIRs were taken into account for producing this plot. Number of RIRs for each quantity combination: C1: 492, C2: 200, C3: 196, C4: 188, C5: 152 .

measurements revealed that the high reverberation time can be attributed to very non-uniform distributions of the absorptive material. For example, in room configuration \#171, all of the 50 absorption wedges were placed in front of the window, thus leaving the other three walls acoustically completely untreated. Consequently, reverberation times between $1.14 \mathrm{~s}$ and $1.62 \mathrm{~s}$ were measured, depending on which of the four loudspeakers acted as the excitation source. The decay curve fitting was inspected manually for these cases and no fitting errors were observed. In contrast, the 50 absorption wedges in room configuration \#166 were equally distributed at three of the four walls. In this case, the reverberation time values ranged between $0.48 \mathrm{~s}$ and $0.62 \mathrm{~s}$.

Secondly, Figure 7 shows distributions with two distinct maxima for the furniture combination $\mathrm{C} 5$ in the $2 \mathrm{kHz}$ and $4 \mathrm{kHz}$ frequency bands. The corresponding measurements featured two containers, and lower reverberation time values were measured for the room configurations, in which absorption wedges were standing on top of the containers. In contrast, higher reverberation times were observed for room configurations, in which no absorption wedges were placed on top of the containers or in which the absorption wedges were lying on them, thus not extending to higher levels in the room.

While it is out of the scope of this paper to conduct an in-depth analysis as in [1], [2], we could still reproduce the variability of reverberation time which was already observed in the above studies. In future studies, the present dataset could be used to extend current research and investigate the effect of 
furniture-caused scattering on room acoustic parameters with a large number of real-world measurements.

\section{CONCLUSION}

We have presented the Motus dataset, which is a new dataset of higher-order Ambisonic room impulse responses measured in a single room with varying furniture. The dataset contains responses of 830 unique room configurations, which differ regarding the quantity and placement of furniture objects inside the room. 3D models and $360^{\circ}$-photographs are available for all room configurations. The measurement setup consisted of four loudspeakers and one 4th-order microphone array and remained static throughout the dataset acquisition.

We demonstrated the usage of the presented dataset with a reverberation time analysis. Apart from some exceptions, our observations are in line with the general trend predicted from theory, that the reverberation time decreases with an increasing absorption area in the room. The exceptions can be attributed to room configurations with highly non-uniform distributions of absorptive materials or errors due to insufficiently robust decay fitting algorithms. Our analysis indicates that an unsupervised room decay analysis on such large datasets requires fitting algorithms and toolboxes, which are more robust to phenomena like multi-slope decays. Moreover, we showed with a large number of measurements that furniture placement can have a considerable effect on the reverberation time of a room.

So far, we have not used the full potential of our dataset because the analyses of this paper were only based on the omnidirectional channel of the Ambisonic stream. However, future research may benefit from the additional information provided by the higher-order sound-field capture. For example, our dataset may be valuable for research in room acoustics and for conducting more in-depth analyses of absorber placement in rooms. Due to the large number of measurements and their variability, the dataset can also support the development and evaluation of decay analysis and fitting algorithms. The availability of matching 3D models for every measured room configuration may benefit the evaluation of acoustic room simulation software. Furthermore, the dataset may support research in source separation and enhancement or virtual acoustic rendering.

\section{Availability And COMPanion Page}

The dataset is available online [20]. A companion page, including audio examples and flip-book-inspired animations of the measured room configurations, can be found at:

$$
\begin{aligned}
& \text { http://research.spa.alto.fi/ } \\
& \text { publications/papers/i3da21-motus/ }
\end{aligned}
$$

\section{ACKNOWLEDGMENT}

The authors would like to thank Karolina Prawda and Thomas McKenzie for their helpful comments on the first draft of the manuscript.

The project has received funding from the Academy of Finland, project no. 317341.

\section{REFERENCES}

[1] J. Cucharero, T. Hänninen, and T. Lokki, "Influence of Sound-Absorbing Material Placement on Room Acoustical Parameters," Acoustics, vol. 1, no. 3, pp. 644-660, 2019.

[2] S. R. Bistafa and J. S. Bradley, "Predicting reverberation times in a simulated classroom," J. Acoust. Soc. Am., vol. 108, no. 4, pp. 17211731, 2000.

[3] M. Kuster, D. d. Vries, E. M. Hulsebos, and A. Gisolf, "Acoustic imaging in enclosed spaces: Analysis of room geometry modifications on the impulse response," J. Audio Eng. Soc., vol. 116, no. 4, pp. 21262137, 2004.

[4] H. Kuttruff, Room Acoustics, 4th ed. London, UK: Spon Press, 2000.

[5] W. Yang and J. S. Bradley, "Effects of room acoustics on the intelligibility of speech in classrooms for young children," The Journal of the Acoustical Society of America, vol. 125, no. 2, pp. 922-933, 2009.

[6] B. Rafaely, Fundamentals of Spherical Array Processing, ser. Springer Topics in Signal Processing. Berlin, Heidelberg; Germany: Springer, 2015, no. 8.

[7] V. Pulkki, S. Delikaris-Manias, and A. Politis, Parametric TimeFrequency Domain Spatial Audio, 1st ed. Hoboken, NJ, USA: Wiley, 2018.

[8] F. Zotter and M. Frank, Ambisonics - A Practical 3D Audio Theory for Recording, Studio Production, Sound Reinforcement, and Virtual Reality, ser. Springer Topics in Signal Processing. Cham, Switzerland: Springer Nature, 2019, no. 19.

[9] L. McCormack, S. Delikaris-Manias, A. Politis, D. Pavlidi, A. Farina, D. Pinardi, and V. Pulkki, "Applications of Spatially Localized ActiveIntensity Vectors for Sound-Field visualization," J. Audio Eng. Soc., vol. 67, no. 11, pp. 840-854, 2019.

[10] S. Tervo and A. Politis, "Direction of Arrival Estimation of Reflections from Room Impulse Responses Using a Spherical Microphone Array," IEEE/ACM Trans. Audio, Speech, Language Process., vol. 23, no. 10, pp. 1539-1551, 2015.

[11] E. Hadad, F. Heese, P. Vary, and S. Gannot, "Multichannel audio database in various acoustic environments," in 14th Int. Workshop Acoust. Sig. Enhancement (IWAENC). Antibes - Juan les Pins, France: IEEE, 2014, pp. 313-317.

[12] R. Stewart and M. Sandler, "Database of omnidirectional and B-format room impulse responses," in Int. Conf. Acoust., Speech, Sig. Proc. (ICASSP). Dallas, TX, US: IEEE, 2010, pp. 165-168.

[13] P. Stade, B. Bernschütz, and M. Rühl, "A Spatial Audio Impulse Response Compilation Captured at the WDR Broadcast Studios," in 27th Tonmeistertagung (TMT). Cologne, Germany: VDT, 2012, pp. 1-17.

[14] J. Y. C. Wen, N. D. Gaubitch, E. A. P. Habets, T. Myatt, and P. A. Naylor, "Evaluation of speech dereverberation algorithms using the MARDY database," in 10th International Workshop on Acoustic Echo and Noise Control (IWAENC). Paris, France: ENST, 2006, pp. 1-4.

[15] M. Jeub, M. Schäfer, and P. Vary, "A binaural room impulse response database for the evaluation of dereverberation algorithms," in 16th International Conference on Digital Signal Processing. Santorini, Greece: IEEE, 2009, pp. 1-5.

[16] H. Kayser, S. D. Ewert, J. Anemüller, T. Rohdenburg, V. Hohmann, and B. Kollmeier, "Database of Multichannel In-Ear and Behind-theEar Head-Related and Binaural Room Impulse Responses," EURASIP J. Adv. Signal Process., vol. 2009, no. 1, pp. 1 - 10 (Article No. 298 605), 2009.

[17] S. V. A. Garí, B. Sahin, D. Eddy, and M. Kob, "Open Database of Spatial Room Impulse Responses at Detmold University of Music," in 149th Convention of the Audio Engineering Society. Online Conference: AES, 2020, pp. 1-5.

[18] A. Neidhardt, "Data set: BRIRs for position-dynamic binaural synthesis measured in two rooms," in 5th International Conference on Spatial Audio (ICSA). Ilmenau, Germany: VDT, 2019, pp. 165 - 169.

[19] A. Farina, "Simultaneous Measurement of Impulse Response and Distortion with a Swept-Sine Technique," in 108th Convention of the Audio Engineering Society. Paris, France: AES, 2000, pp. 1-23.

[20] G. Götz, S. J. Schlecht, and V. Pulkki, "Motus: A dataset of higher-order Ambisonic room impulse responses and 3D models measured in a room with varying furniture," Jun. 2021. [Online]. Available: https://doi.org/10.5281/zenodo.4923187

[21] M. Karjalainen, P. Antsalo, A. Mäkivirta, T. Peltonen, and V. Välimäki, "Estimation of Modal Decay Parameters from Noisy Response Measurements," J. Audio Eng. Soc., vol. 50, no. 11, pp. 867-878, 2002. 
[22] M. Berzborn, R. Bomhardt, J. Klein, J.-G. Richter, and M. Vorländer "The ITA-Toolbox: An Open Source MATLAB Toolbox for Acoustic Measurements and Signal Processing," in 43rd Jahrestagung für Akustik (DAGA). Kiel, Germany: DEGA, 2017, pp. 222-225.

[23] Institute of Sound Recording (University of Surrey), "IoSR Matlab Toolbox," [Online]. Available: https://github.com/IoSR-Surrey/ MatlabToolbox, 2017, Last accessed: 14 December 2020.

[24] ISO 3382-2, "Acoustics - Measurement of room acoustic parameters Part 2: Reverberation time in ordinary rooms," International Organization for Standardization (ISO), Geneva, Switzerland, Standard, 2008. 